\title{
Entering a new phase
}

\section{B. W. Batterman}

Crystallographers recording the diffraction patterns of X-rays scattered by crystals are losing vital information - the phase of the X-rays. On page 232 of this issue, Shen and Colella offer a practical solution to the phase problem. If it can be solved, then the intensity of the Bragg reflections, which appear as spots on diffraction patterns, can be used to determine unambiguously the position and type of all the atoms that make up the fundamental unit from which the crystal is built.

The intensity of a wave producing the Bragg reflection is proportional to the square of the amplitude of its sinusoidal oscillation. When an X-ray wave strikes the film, the blackening produced is proportional to the intensity of the wave, and from this the amplitude can easily be determined. The phase of the sine wave (where it goes through zero in time with respect to the other Bragg spots) is lost, and to reconstruct the three-dimensional crystal from its two-dimensional diffraction pattern the phase and the amplitude of each Bragg reflection are needed.

Analogous to the phase problem is the difference between the holographic image of an object and its standard camera representation on a sheet of film. The film image clearly lacks information available from the hologram. The latter is a full three-dimensional representation of the object, so that one can see all sides of it; the former gives the same limited twodimensional information irrespective of how it is viewed. The three-dimensional character of the hologram derives from the fact that the relative phase of the light from each point on the object is recorded.

The problem is how to record intensity and phase information on film that is sensitive only to intensity. To do this requires changing the phase information into intensity information, achieved by comparing the scattered waves with an unscattered reference wave. The interference pattern obtained by mixing the two travelling waves is a series of peaks and troughs that are stationary in time producing a moiré-like pattern of fringes whose intensities depend on the amplitude of the scattered wave and its phase relative to the reference. Moving the object changes the phase, so that the pattern of fringes will change. The intensity and phase of each object point are simultaneously recorded by the film.

A more conventional approach to phasing is a technique called isomorphous replacement. In this case, the reference phase is obtained from the scattering of a heavy atom inserted in the structure, preferably with a minimal perturbation of the surrounding atomic positions. Isomorphous replacement is an invasive technique with limited applicability.

Statistical phasing of the direct method variety was pioneered by Karle and Hauptmann who were awarded the 1986 Nobel prize for their achievements. Their method takes account of certain relationships between phases of reflection derived from the global condition that the electron density must be everywhere positive. Although a powerful technique for small molecule structures it has not been successfully applied to large proteins.

For X-ray diffraction, Shen and Colella transform the phase of a particular Bragg reflection to an intensity variation by simultaneously exciting a second Bragg reflection that acts as a reference wave. Multiple Bragg excitations have been used by others (Colella, R. Acta crystallogr. A30, 413; 1974 and Post, B. Phys. Rev. Lett. 39, 760; 1977) to obtain the phase of a given reflection. In general only crystals of relatively simple structure and extremely high perfection have been used to demonstrate the ability to measure phase. Few crystals are highly perfect and the most interesting have complex structures.

Shen and Colella show that even with crystals of poor perfection, multiple-beam excitation can be used to obtain the relative phase of a given Bragg reflection with respect to a reference reflection. Their method, demonstrated with a crystal of benzil, involves the rather straightforward

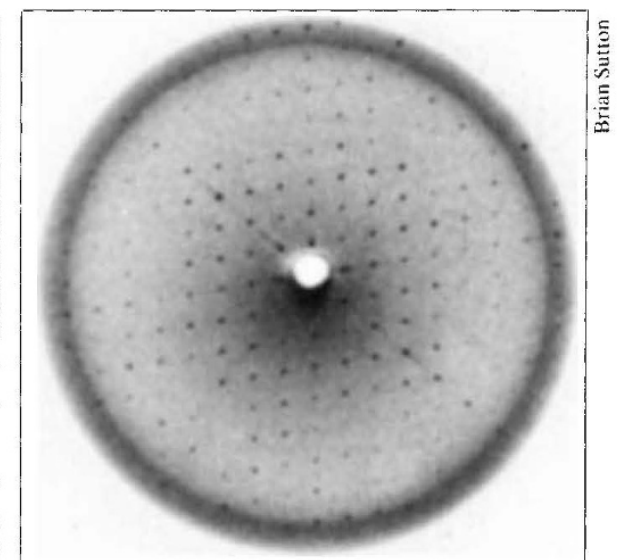

$X$-ray diffraction pattern of $\beta$-lactamase II. Could the new method solve its structure? recording of the intensity of the reflection as the reference beam is brought into Bragg diffracting conditions. The observed intensity displays an asymmetrical peaking as the reference beam is continuously swept through its Bragg condition while maintaining Bragg reflection of the primary beam. The phase of the reflection is found by measuring the relative intensity difference between the left and right wings of the asymmetrical peak.

Shen and Colella have solved the phase problem using a relatively simple technique, the limits of which will no doubt be pursued by the crystallographers. Key questions to be answered are: can a sufficient number of reflections be phased to solve any particular structure? How imperfect can the crystal be? What is the upper limit to the size of the unit cell for which the technique is effective? The answers will prove the efficacy of Shen and Colella's technique.

B. W. Batterman is at the Cornell High Energy Synchroton Source, Cornell University, Ithaca, New York 14853, USA.

\section{Mathematics}

\section{Geometry goes non-commutative}

\section{John D.S. Jones}

A VIEw sometimes expressed is that the basic objects of study in mathematics are numbers, geometry and physical phenomena, and that the basic methods with which one studies these are algebra and analysis. Most mathematicians would greet this assertion with something like "Yes, but what about. . . ."Nonetheless there is an element of truth in the idea. Recently, Alain Connes has performed the mathematical equivalent of the threecard trick: he has found a rather unexpected way of applying some sophisticated parts of modern analysis, which have their origins in mathematical physics, to geometrical problems (Pub. Math. IHES 62, 41-144; 1985). One exciting aspect of his approach is the way he draws on experience and intuition from different areas of mathematics and makes links between them. Ideas from topology and geometry are mixed with ideas from analysis and mathematical physics to the benefit of all. It is intriguing that one of the things to come out of this mixture is the introduction of a new algebraic technique, cyclic cohomology.

To anyone with a little mathematical knowledge, the title of Connes' artciles (Non-Commutative Differential Geometry $I$ and $I I)$ may sound ridiculous. After all, the commutative law is the rule of multiplication, $a b=b a$. There are algebraic systems that do not obey this law, for example the multiplication of matrices, but at first sight such systems do not seem 